\title{
Simulation assisted ultrasonic defect characterization in heavy rotor forgings
}

\author{
Karl T. Fendt ${ }^{1,2}$, Hubert Mooshofer ${ }^{1}$, Stefan J. Rupitsch ${ }^{2}$, Helmut Ermert ${ }^{2}$ \\ ${ }^{1}$ Siemens AG, Munich Germany \\ karl.fendt@fau.de \\ ${ }^{2}$ Chair of Sensor Technology, Friedrich-Alexander-Universität Erlangen-Nürnberg, Erlangen Germany
}

\begin{abstract}
For heavy rotor components of turbines and generators, rigorous ultrasonic inspection during the manufacturing process is mandatory. Its purpose lies in detection and classification of flaws, with the results providing a basis for fracture mechanical predictions. In this contribution, a new method for classifying ultrasonic indications is presented: Ultrasonic data is reconstructed by means of Synthetic Aperture Focusing Technique (SAFT), and indication positions are extracted. Next, we perform numerical simulations containing model reflectors at the determined positions. The defect model parameters are iteratively modified to minimize the difference between measured and simulated inspection data. This leads to a set of defect parameters which best match the flaw indications. The method is applied to laboratory and forging blank inspection data, where it correctly determines defect characteristics.
\end{abstract}

\section{Introduction}

Ultrasonic nondestructive inspection is one of the most commonly used techniques to ensure structural integrity. In the manufacturing of heavy turbo machinery such as turbines or generators, rotor components are considered safety-critical due to multiple critical failures over the past decades [1]. Therefore, all forgings for rotor components are rigorously tested, typically using multi-angle pulse-echo ultrasonic inspection [2]. In addition to identifying parts that do not satisfy specifications, results of ultrasonic inspection also provide a basis for lifetime assessment. Flaw positions and sizes are used in conjunction with component characteristics (e.g., material, geometry) to conduct fracture mechanical simulations, resulting in failure probability as a function of time [3].

There is interest in the application of ultrasonic reconstruction algorithms such as the Synthetic Aperture Focusing Technique (SAFT) to forging inspection data [4], to increase signal-to-noiseratio (SNR) and resolution. Large defects can be characterized by the size of the ultrasonic indication, a method which directly benefits from the refocusing effect of SAFT. Exemplary, Fig. 1 shows the 3D SAFT reconstruction of a turbine shaft containing a long flaw. Measuring the largest dimension of the indication, which is oriented almost parallel to the rotor axis, yields an approximate length of $14 \mathrm{~cm}$.

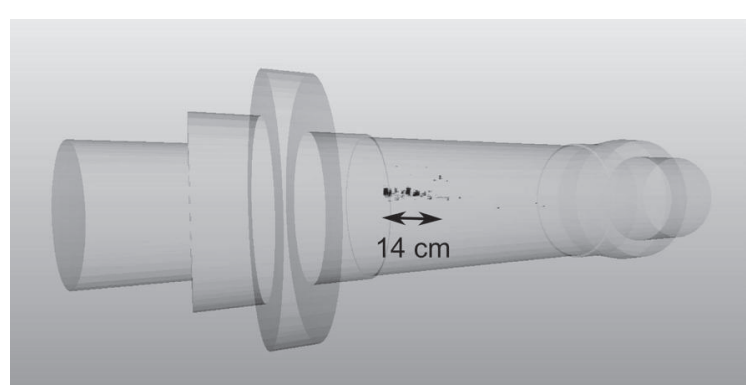

Fig.1. SAFT $3 D$ visualization of a turbine shaft inspection, containing a large discontinuity. Black voxels represent reconstruction points at which intensities above a registration value are observed. The indication length is approximately $14 \mathrm{~cm}$.

Small flaws, however, cannot be quantified by this technique due to increasing dominance of imaging characteristics. This is illustrated in Fig. 2 , where reconstruction results of two machined reflectors differing only in size are shown. The two flat-bottom holes with nominal diameters of 3 and $1.5 \mathrm{~mm}$ result in ultrasonic indications with lateral $6-\mathrm{dB}$ widths of 4 and $3.7 \mathrm{~mm}$, respectively, which are unsuitable measures for flaw quantification. 


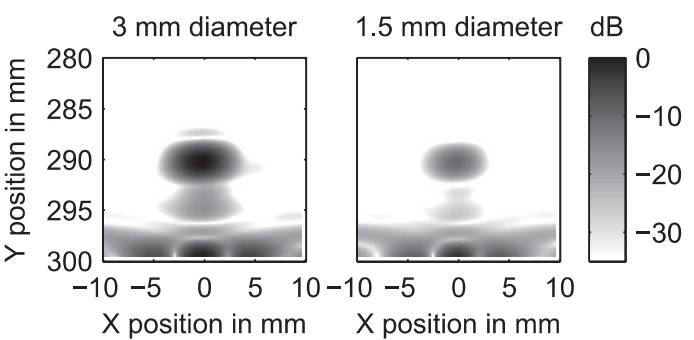

Fig. 2. SAFT reconstructions of differently sized machined reflectors. The left indication is from a 3 $\mathrm{mm}$, and the right from a $1.5 \mathrm{~mm}$ diameter flatbottom hole, both inspected in the same scan. Although intensity differs, the indications $-6 \mathrm{~dB}$ lateral width shows only a slight decrease from 4 to $3.7 \mathrm{~mm}$.

In this contribution, a method to further characterize such small indications is developed. One approach to identify unknown physical quantities is to find a simulation parameterization which yields synthetic results that match the measured data [5]. We apply this concept here, using a semi-analytical elastodynamic simulation in Kirchhoff approximation. Initial defect model parameters are determined by SAFT reconstruction results and optimized by means of an iteratively regularized Gauss-Newton method. The following sections describe the individual components of the proposed method in detail, followed by experimental results and their discussion.

\section{SAFT}

The first applications of synthetic aperture focusing to ultrasonic imaging date back to the 1970s [e.g., 6]. It increases resolution and improves SNR by incorporating signals from multiple transducer locations into the reconstruction, thus forming a synthetic aperture. One of the simplest implementations, the delay-and-sum algorithm, consists of coherent summation of RF data $a(\mathbf{r}, t)$, delayed according to the distance between transducer positions $\mathbf{r}=(x, y, z)$ and reconstruction point $\mathbf{r}^{\prime}$. In order to obtain a demodulated image, the complex valued analytic signal

$$
a^{+}(\mathbf{r}, t)=a(\mathbf{r}, t)+j H\{a(\mathbf{r}, t)\}
$$

of the RF data is used here. In Equ. 1, $H\{\cdot\}$ denotes the Hilbert transform. Additionally, the image function $I\left(\mathbf{r}^{\prime}\right)$ described in Equ. 2 contains a weighting factor $\alpha\left(\mathbf{r}, \mathbf{r}^{\prime}\right)$ to suppress measurement points which cannot physically contribute to the reconstruction point (i.e., no significant signal intensity is transmitted in the corresponding direction). $M$ is the entirety of all measurement positions, i.e., the synthetic aperture.

$$
I\left(\mathbf{r}^{\prime}\right)=\frac{\left|\sum_{\mathbf{r} \in M} \alpha\left(\mathbf{r}, \mathbf{r}^{\prime}\right) a_{i}^{+}\left(\mathbf{r}, \frac{2\left|\mathbf{r}-\mathbf{r}^{\prime}\right|}{c}\right)\right|}{\sum_{\mathbf{r} \in M} \alpha\left(\mathbf{r}, \mathbf{r}^{\prime}\right)}
$$

We then identify indications in the reconstructed data, which are to be further classified. Their maxima locations provide the initial parameterization for model based inversion.

\section{Simulation}

To classify flaws by the proposed method, a suitable simulation environment is necessary. Ultrasonic inspections can be simulated by different methods, including finite difference time domain and finite element methods, which both accurately model most wave phenomena. However, due to potentially long propagation paths and the high number of simulations required in the proposed method, simulations based on discretization of the entire problem domain are unsuitable. Instead, a fast, semianalytical simulation [7] is employed here. In this approach, linearity is assumed and the output signal is obtained by convolution of a pulse form $\mathrm{u}(\mathrm{t})$ with transfer functions modeling the transmission, reception and scattering process.

$$
a(t)=u(t) * h_{T}(t) * h_{S}(t) * h_{R}(t)
$$

The transmission $\left(h_{T}(t)\right)$ and reception transfer function $\left(h_{R}(t)\right)$ are calculated by means of elastodynamic farfield approximation formulas. For the case of pulse-echo measurements, reciprocity can exploited and consequently, we are able to use the same transfer function for both processes.

Scattering is simulated under the Kirchhoff approximation, which is frequently applied in the context of ultrasonic nondestructive testing (NDT). The flaw surface is modeled as a secondary ultrasonic source and the resulting fields are calculated according to reflection coefficients that would occur on strain-free, plane surfaces (the tangent plane for every surface point).

Using this semi-analytical method, a simulation model resembling the measurement configuration is constructed and flaw models are added in accordance with the results of the SAFT processing step. Here, the defect geometry model is constrained to plane circular 
flaws, but the method can be applied to different geometries. The initial parameter set, especially the defect size, can only be set approximately, and hence the optimization technique described in the next section is employed to properly characterize flaws.

\section{Iteratively regularized Gauss-Newton method}

An iteratively regularized Gauss-Newton algorithm is utilized to solve the ill-posed problem of finding the defect parameter set $\mathbf{p}$ for which the simulation results $a_{S}(\mathbf{p}, \mathbf{r}, t)$ best fit the measurement data $a(\mathbf{r}, t)$. This is achieved by minimization of the following functional [8]

$$
\begin{aligned}
& \left\|\mathbf{R}^{\prime}\left(\mathbf{p}_{n}\right) \mathbf{s}_{n}+\mathbf{R}\left(\mathbf{p}_{n}\right)\right\|_{2}^{2}+ \\
& \xi_{n}\left\|\mathbf{p}_{n}-\mathbf{p}_{0}\right\|_{2}^{2} \rightarrow \text { min, }
\end{aligned}
$$

where $\xi_{n}$ is a positive valued weighting parameter of the penalty term that decreases to zero with increasing number of iterations $n$. $\mathbf{R}\left(\mathbf{p}_{n}\right)$ denotes the residual, or difference between simulation and measurement, and $\mathbf{R}^{\prime}\left(\mathbf{p}_{n}\right)$ the corresponding Jacobian. The parameter update vector $\mathbf{s}_{n}$ can then be calculated by

$$
\begin{aligned}
& \mathbf{s}_{n}=\left[\mathbf{R}^{\prime}\left(\mathbf{p}_{n}\right)^{T} \mathbf{R}^{\prime}\left(\mathbf{p}_{n}\right)+\xi_{n} \mathbf{I}\right]^{-1} \\
& {\left[\mathbf{R}^{\prime}\left(\mathbf{p}_{n}\right)^{T} \mathbf{R}\left(\mathbf{p}_{n}\right)+\xi_{n}\left(\mathbf{p}_{n}-\mathbf{p}_{0}\right)\right]}
\end{aligned}
$$

Using this algorithm, the following parameters are optimized: Size and location of the disc shaped model flaw, as well as two angles determining the ultrasound transducers beam axis.

\section{Experimental results}

In this section, we apply the proposed defect quantification method to ultrasonic inspection datasets. The first specimen, a turbine disk forging blank, is a steel cylinder with a diameter of approximately $1.46 \mathrm{~m}$ and $0.48 \mathrm{~m}$ length. It contains a large inner bore of $0.246 \mathrm{~m}$ diameter. The artificial reference flaw of interest is an $18 \mathrm{~mm}$ long flat-bottom hole of $1 \mathrm{~mm}$ diameter, drilled from the inner bore (see Fig.3).

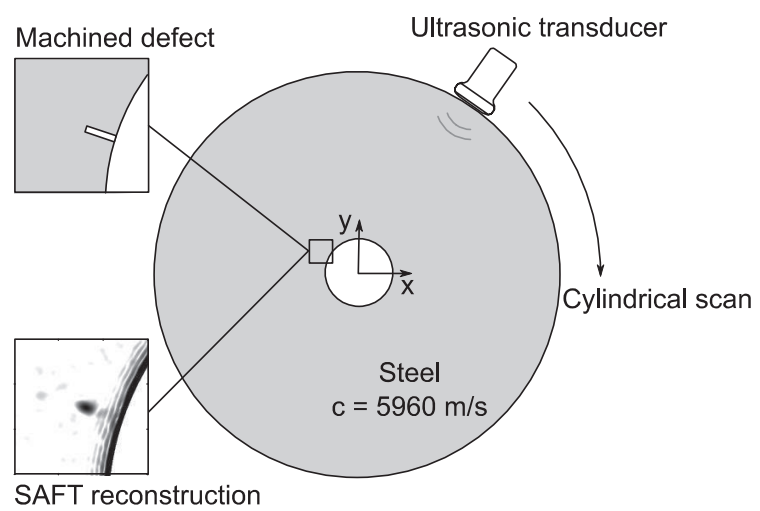

Fig.3. Schematic of the forging blank specimen and inspection setup, including a close-up of the machined defect and corresponding SAFT reconstruction. The reflector is a $1 \mathrm{~mm}$ diameter flatbottom hole of $18 \mathrm{~mm}$ length, machined from the large inner bore.

Inspection data consists of a $2.25 \mathrm{MHz}$ cylindrical scan with an angular resolution of $0.39^{\circ}$ and spatial resolution of $5 \mathrm{~mm}$, performed in contact mode. The sampling frequency of the recorded RF data is $12.5 \mathrm{MHz}$.

SAFT reconstruction of the region of interest containing the machined reflector yields approximate flaw positions (see Tab. 1). At these positions, the initial defect model - a disc shaped reflector with $2 \mathrm{~mm}$ diameter - is added to a simulation parameterized in accordance with the measurement setup (longitudinal wave speed of $5960 \mathrm{~m} / \mathrm{s}$ ). The reflector disc axis is oriented in radial direction.

Results obtained by the optimization procedure are listed in Tab. 1. Defect size is reconstructed to $1.03 \mathrm{~mm}$ (1 $\mathrm{mm}$ nominal), and $18.2 \mathrm{~mm}$ distance from the large inner bore (nominal length of $18 \mathrm{~mm}$ ).

Tab. 1: Simulation model parameters for the forging blank inspection

\begin{tabular}{|c|c|c|}
\hline Parameter & $\begin{array}{c}\text { Initial } \\
\text { values }\end{array}$ & $\begin{array}{c}\text { Optimization } \\
\text { result }\end{array}$ \\
\hline Defect x position & $-133 \mathrm{~mm}$ & $-133.7 \mathrm{~mm}$ \\
\hline Defect y position & $-45 \mathrm{~mm}$ & $-45.3 \mathrm{~mm}$ \\
\hline Defect z position & $242 \mathrm{~mm}$ & $241.9 \mathrm{~mm}$ \\
\hline Defect diameter & $3 \mathrm{~mm}$ & $1.03 \mathrm{~mm}$ \\
\hline $\begin{array}{c}\text { Transducer tilt } \\
\text { angle in z }\end{array}$ & $0^{\circ}$ & $3.3^{\circ}$ \\
\hline $\begin{array}{c}\text { Transducer tilt } \\
\text { angle in x-y }\end{array}$ & $0^{\circ}$ & $0.3^{\circ}$ \\
\hline
\end{tabular}


To determine the quality of the proposed approach, a comparison between measurement data containing the defect echo signal and the corresponding residual after optimization can be made. This comparison is qualitatively illustrated in Fig. 4, where the original defect signal appears to be almost entirely eliminated in the residual.

Quantitatively, it makes sense to restrict the evaluation to parts of the residual with a significant energy level in the synthetic signal. Discarding data points where the envelope amplitude of the simulated data decreases below $20 \%$ of its maximum, the residual signal energy amounts to $37.5 \%$ of the original measurement signal energy.
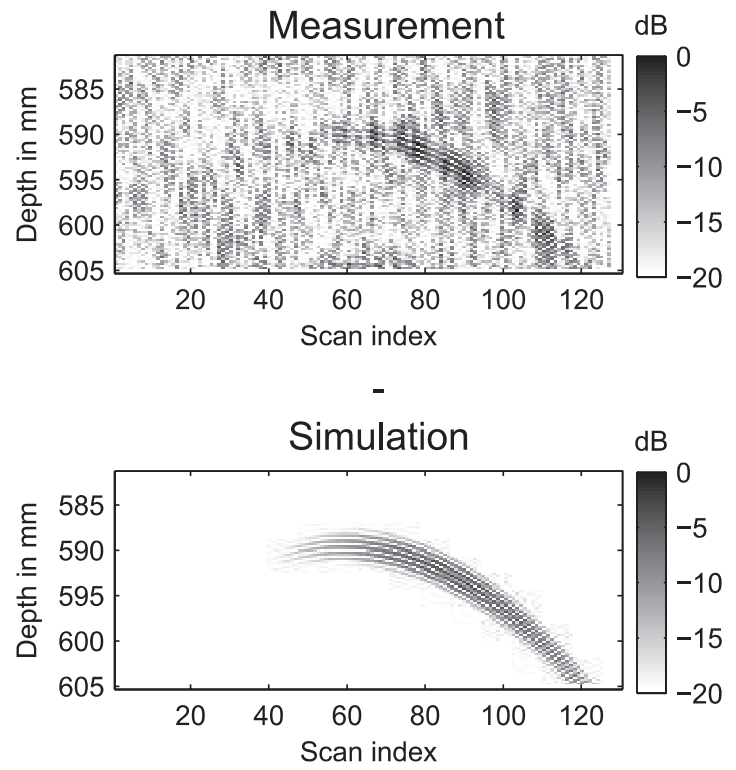

$$
=
$$

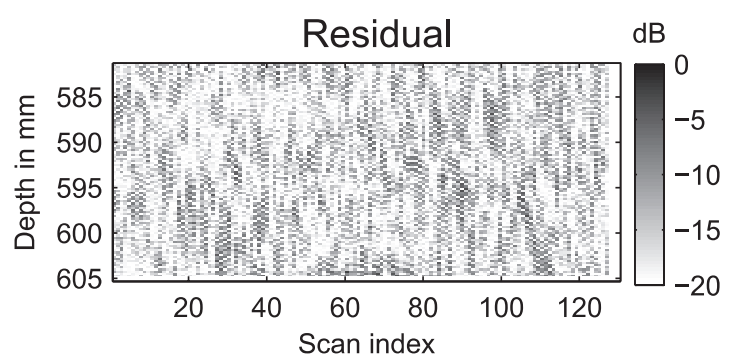

Fig.4. A comparison between measured flaw echo signal, synthetic signal after optimization and residual, in which virtually no defect signal is distinguishable.

To demonstrate the benefit of increased resolution achieved by the incorporation of SAFT in the proposed procedure, we investigated a second configuration. The specimen is a $300 \times 200 \times 100 \mathrm{~mm}^{3}$ steel block containing two flat-bottom holes of $20 \mathrm{~mm}$ length and spaced $10 \mathrm{~mm}$ apart. It was inspected in contact mode over the surface opposed to the machined defects with a $2 \mathrm{MHz}$, $10 \mathrm{~mm}$ diameter transducer. We performed a planar scan with a step size of $1 \times 1 \mathrm{~mm}^{2}$ and recorded RF data at $50 \mathrm{MHz}$ sampling frequency.

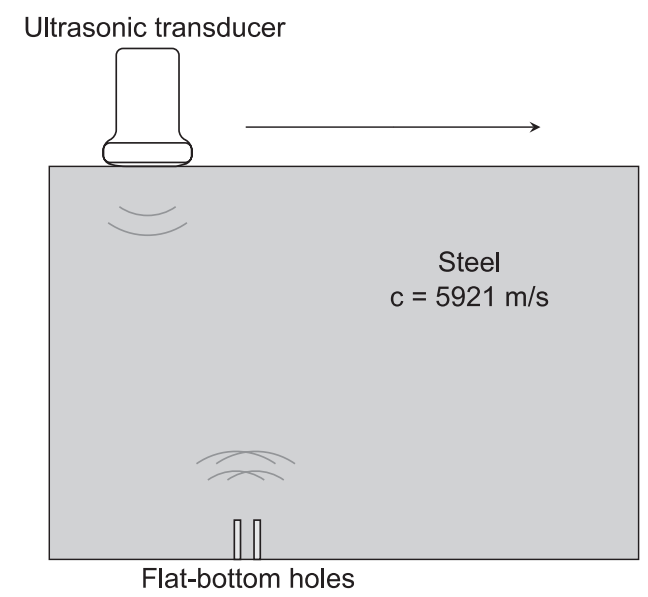

Fig.5. Schematic of the second considered specimen, containing two $20 \mathrm{~mm}$ long flat-bottom holes of $2 \mathrm{~mm}$ diameter, in $10 \mathrm{~mm}$ distance.

Fig. 6 shows a single slice of the ultrasonic dataset. Due to interference between the reflected signals, the two reflectors cannot be resolved in the B-scan representation, making correct identification and localization impossible.

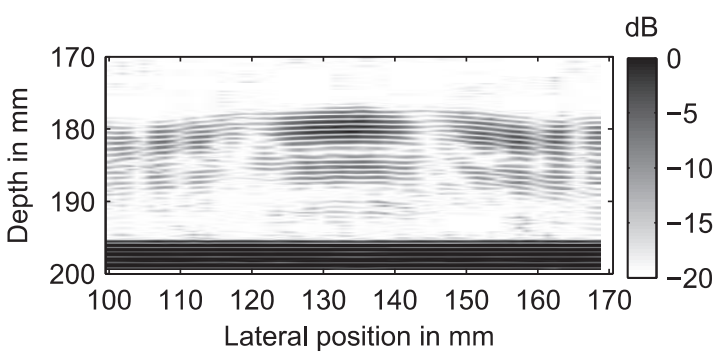

Fig.6. B-Scan image of the region of interest containing the two neighboring defects (see Fig. 5).

After SAFT reconstruction, the two reflectors are clearly resolved, and the local indication maxima are located at the correct lateral positions.

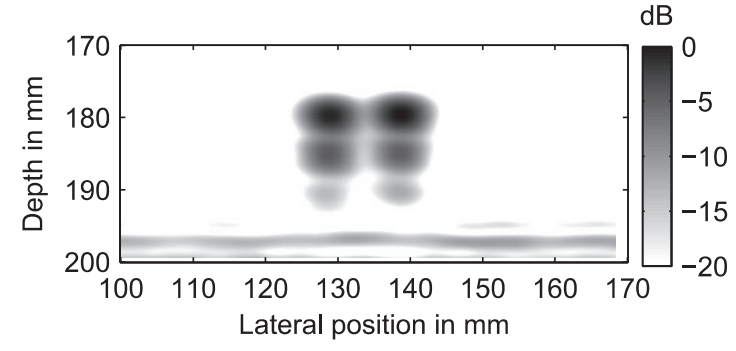

Fig.7. SAFT image of the same region as in Fig.6. Due to the increased lateral resolution, the two 
defects are clearly distinguishable and can be correctly located.

Using the two maxima locations, initial flaw models of $3 \mathrm{~mm}$ diameter discs with orientation parallel to the inspection surface are considered. Location, defect size and transducer tilt are optimized, yielding the following quantities: A $2.1 \mathrm{~mm}$ diameter flaw in $180.1 \mathrm{~mm}$ depth, and a $2.05 \mathrm{~mm}$ diameter flaw in $180.2 \mathrm{~mm}$ depth, in $10.08 \mathrm{~mm}$ distance from each other. These results fit well the nominal configuration of two defects. Transducer tilt was determined to below $0.5^{\circ}$ in both directions.

\section{Conclusion}

In this contribution, a procedure for characterizing flaws by means of ultrasonic inspection was proposed. It combines the Synthetic Aperture Focusing Technique for flaw identification with a simulation based optimization method for subsequent quantification. We tested the procedure on inspection data of a large cylindrical forging blank containing a machined flaw. Both the flaw location and geometric dimension were accurately reconstructed. To demonstrate the advantage of incorporating SAFT in the procedure a second setup was considered, where a proper defect identification would not be possible without SAFT.

\section{References}

[1] J. Vrana, et al. "Evolution of the Ultrasonic Inspection Requirements of Heavy Rotor Forgings over the Past Decades", Review of Progress in QNDE 29, 1623-1630 (2010); doi: 10.1063/1.3362263

[2] Stahlinstitut VDEh - Gemeinschaftsausschuss schwere Schmiedestuecke, "Ultrasonic testing of steel forgings to stringent standards, in particular for components in turbine and generator systems - SEP 1923", STAHL-EISEN-Pruefblaetter des Stahlinstitut VDEh (2009)

[3] R. Viswanathan, Damage mechanisms and life assessment of high temperature components. ASM international (1989)

[4] R. Böhm, W. Heinrich: „Stoersignalminderung bei der Ultraschallprüfung durch ein Auswerteverfahren in Anlehnung an die Synthetic Aperture Focussing Technique (SAFT)“, DGZfPJahrestagung St. Gallen (2008)

[5] S. J. Rupitsch, F. Wolf, A. Sutor, and R. Lerch. "Reliable modeling of piezoceramic materials utilized in sensors and actuators." Acta Mechanica 223, no. 8, 1809-1821 (2012); doi: $10.1007 / \mathrm{s} 00707-012-0639-7$

[6] H. Ermert and R. Karg. "Multifrequency acoustical holography" IEEE Transactions on Sonics and
Ultrasonics 26, 279-286 (1979); doi: 10.1109/TSU.1979.31101

[7] K.-J. Langenberg, R. Marklein, and K. Mayer, Ultrasonic Nondestructive Testing of Materials: Theoretical Foundations. CRC Press, (2012)

[8] B. Blaschke, A. Neubauer, and O. Scherzer, "On convergence rates for the iteratively regularized Gauss-Newton method," IMA Journal of Numerical Analysis, vol. 17, no. 3, 421-436 (1997) 